\title{
Filariasis: A Rare Differential Diagnosis of Cystic Neck Swelling
}

\section{Bihani K, Kamble P, Arora R* and Nagarkar NM}

Department of Otorhinolaryngology, All India Institute of Medical Sciences, India

*Corresponding author: Ripudaman Arora, Department of Otorhinolaryngology, All India Institute of Medical Sciences, Raipur, Chhattisgarh, India, Email: ripuarora@aiimsraipur.edu.in

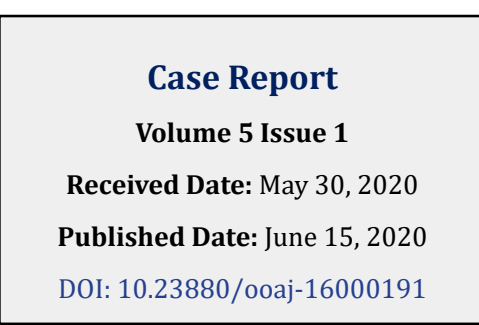

\section{Abstract}

Cystic Neck swelling in adults have a variable differential diagnosis, often either lymphatic, vascular malformation or infective swelling. Filariasis presenting as a cystic isolated neck swelling is a rare unusual presentation of extra lymphatic filariasis. We report a case of 25-year-old male with left supraclavicular swelling for 2 months. Diagnosis was made on detection of the microfilaria on fine needle aspiration cytology and the patient responded excellently to the anti-helminthic drugs with complete resolution in 4 weeks. Although rare filariasis should be considered as a differential diagnosis of aberrant cystic neck swelling as it is one of the important causes of disability and exerts a major impact on health care costs, careful screening of all smears and high index of suspicion, especially in endemic areas are the keys to correct diagnosis.

Keywords: Cystic Neck swelling; Filariasis; Wuchereria bancrofti

Abbreviations: MCG: May-Grunwald Giemsa; PCR: Polymerase Chain Reaction.

\section{Introduction}

A neck mass is a relatively common head and neck presentation in patients presenting to a physician and the differential diagnosis of neck mass is broad and extensive. It includes broad categories of congenital, infectious and neoplastic aetiologies. In the broad spectrum of aetiologies Filariasis a parasitic infection can rarely present as an unusual and rare cause of isolated neck swelling. In tropical and subtropical countries, parasitic infections are unbridled affecting more than 120 million people, 40 million seriously incapacitated and one billion people at risk for infection with $1 / 3$ belong to India [1,2]. Filariasis is caused by two closely related nematode worms, Wuchereria bancrofti and Brugia malayi [3]. The spectrum of illness is wide and varied but asymptomatic microfilaremia is the most common manifestation [4]. The classical features of lymphatic filariasis include lymphangitis, lymphadenitis, hydrocoele, elephantiasis, chyluria, allergic manifestations, and tropical eosinophilia [5]. Filariasis presenting as an isolated neck node involvement is a rare presentation and requires excisional biopsy or fine needle aspiration cytology with imaging studies for definitive diagnosis. Few cases of Filariasis presenting as a head and neck swelling has been documented in the English literature. We thus report a similar such rare case of filariasis presenting as a cystic neck swelling and adding to the importance of diagnosis on fine needle cytology and it is curable with medicines if efficiently treated early.

\section{Case Report}

A 25-year-old male patient presented to the department of ENT and Head \& Neck surgery with complaints of swelling in left supraclavicular region for past 2 months. It was not associated with other positive symptoms of fever, pain, difficulty swallowing or breathing, weight loss decreased appetite, swelling in other sites of body or any ear and nose related complaints. On local examination, there was a smooth swelling size approximately $5 \times 4 \mathrm{~cm}$ in the left supraclavicular region (Figure 1). The skin over was normal. It was soft, cystic in consistency, non-tender, mobile with normal skin temperature. No other neck nodes were positive. Nasal, ear, oral cavity and oropharyngeal examination was normal. Endoscopic laryngeal examination was normal, systemic examination was in normal limits. 


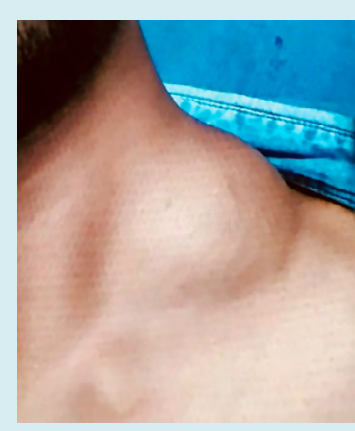

Figure 1: Left supraclavicular swelling shown with arrow.

On routine blood investigations haemoglobin, total and differential leucocyte counts, and peripheral blood smear was normal. The erythrocyte sedimentation rate was normal ruling out chronic inflammation. Further liver and kidney function tests were normal. We further did radiological and cytological investigation to rule out tuberculosis or any other infectious cause. Ultrasonography of neck revealed a well-defined oval shaped heterogenous hypoechoic lesion with internal vascularity on the left supraclavicular region measuring $4.4 \times 4.1 \times 1.5 \mathrm{~cm}$ suggestive of? lymph node or? vascular lesion (Figure 2).

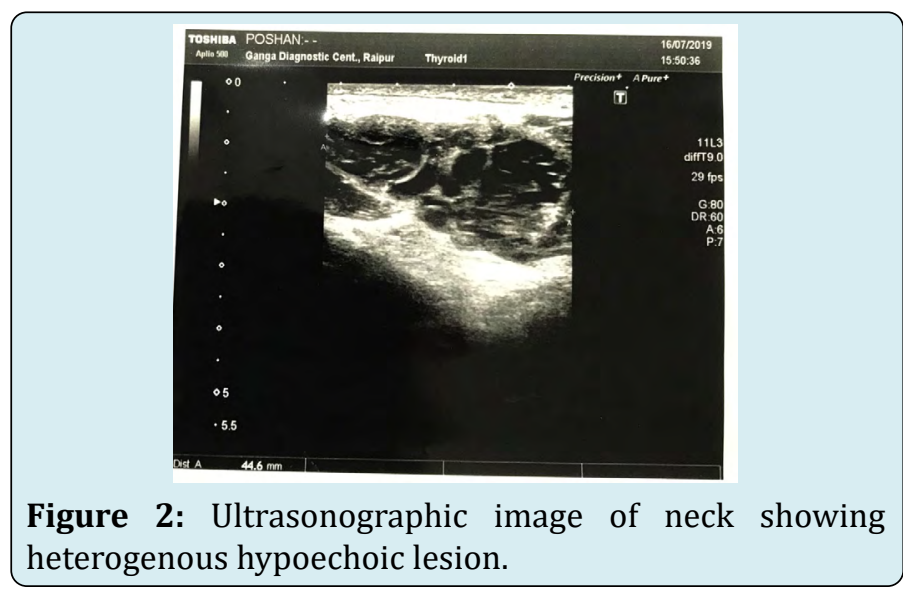

Figure 2: Ultrasonographic image of neck showing heterogenous hypoechoic lesion.

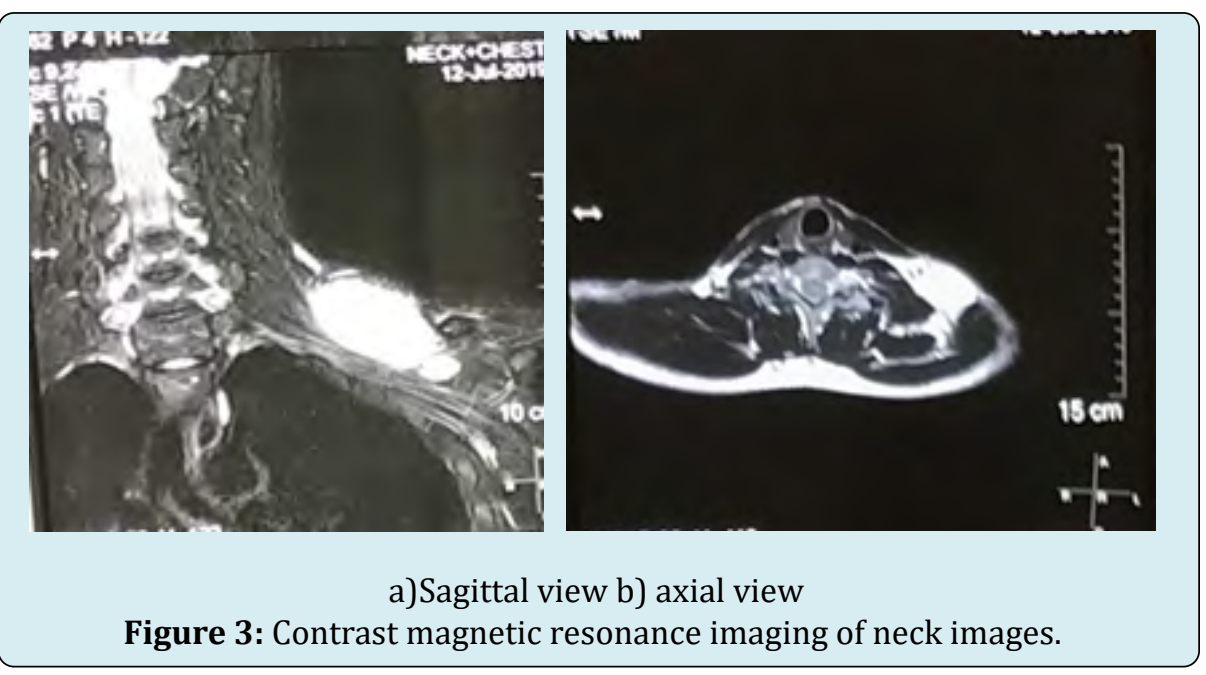

A contrast magnetic resonance imaging was ordered in view of? vascular cystic swelling which revealed a well-defined lobulated mass lesion seen at left retro and supraclavicular region displaying heterogenous hyperintense signal on T2 images, intermediate signal on T1W1 and shows moderate post contrast enhancement. Adjacent subclavian vessels are displaced inferiorly. Branchial plexus division and cord are displaced and compressed posteriorly. Medially it is limited by lateral border of scalene muscle. Measures $5.8 \mathrm{x}$ $5 \mathrm{x} 2.8 \mathrm{~cm}$ in size suggestive of? hemangioma? neoplastic soft tissue mass (Figure 3).

Further, fine needle aspiration cytology yielded $15 \mathrm{ml}$ of thin fluid and on May-Grunwald Giemsa (MGG) the smears shows predominantly mature lymphocytes with adult microfilaria on a fluidic haemorrhagic background with positive anti fialarial antibodies. Subsequently the patient was prescribed diethylcarbamazine and showed complete resolution after 4 weeks with no recurrence or relapse seen after 4 months of follow up (Figure 4).

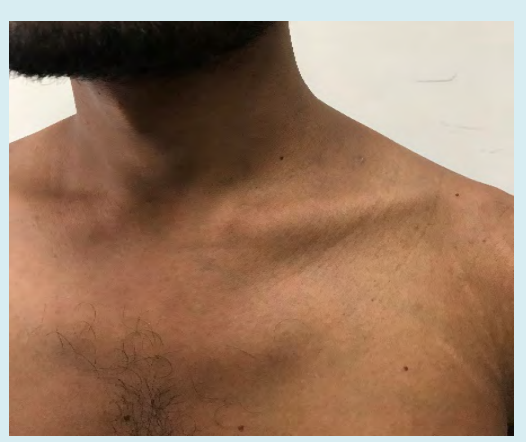

Figure 4: Picture after completion of treatment showing significant reduction of swelling. 


\section{Otolaryngology Open Access Journal}

\section{Discussion}

Filariasis is an endemic disease in India and the most common cause of acquired lymphedema in the world. It is more commonly seen in men and in adult population [6]. Lymphatic filariasis is transmitted by culex mosquito and is mainly caused by two related nematodes, Wuchereria bancrofti and Brugia malai responsible for $90 \%$ and $10 \%$ cases, respectively [7]. The spectrum of illness is wide and varied which includes, pulmonary tropical eosinophilia, acute adeno-lymphangitis, acute and chronic lymphadenitis usually involving lower limb and scrotal swelling but asymptomatic microfilaremia is the most common manifestation. Isolated involvement of neck region is rare and only few cases have been reported. Infectious and neoplastic causes are often suspected like in our case. Fine Needle Aspiration Cytology has emerged as a lowcost out- patient procedure which many a times helps in prompt recognition of the disease especially in unsuspected cases of filariasis. The definitive diagnosis of filariasis depends on demonstration of the parasite either in peripheral blood or in the aspirate or in FNAC [8]. Indirect heamagglutination, ELISA, immunoassays and polymerase chain reaction (PCR) are also helpful in making the diagnosis if microfilaria cannot be found $[9,10]$.

Management of acute filariasis includes anti-helminthic drugs. Diethylcarbamazine $6 \mathrm{mg} \mathrm{kg}-1$ day- 1 for $2-3$ weeks or Ivermectin single dose of $200-400 \mu \mathrm{g} \mathrm{kg}-1$ can be used, but it should be repeated every 6-12 months. Single dose of Ivermectin at 200-400 $\mu \mathrm{g} \mathrm{kg-1}$ plus Albendazole $400 \mathrm{mg}$ are reported to be more effective in some studies [11]. In our case patient reported complete resolution of the symptoms and the swelling with 4 weeks of DEC treatment. Since there has been no chemoprophylaxis, or any radical cure for chronic cases, early diagnosis and treatment is the best option for filariasis.

\section{Conclusion}

Lymphatic filariasis, although rare should be considered as a differential diagnosis in cystic neck swellings, especially in endemic areas with no other positive findings. In our case a diagnosis was made based on FNAC and radiological investigation highlighting the importance of cytological investigation a valuable diagnostic tool to make a diagnosis in maximum cases in the diagnosis of filariasis. Filariasis if misdiagnosed and untreated can result in lymphangitis, lymphatic blockage, lymphedema and disfiguring elephantiasis in the affected organ and its inherent complications. In our case, complete remission of symptoms and swelling seen with antihelminthic drugs was seen, thus emphasizing the importance of early and accurate diagnosis as filariasis is a curable condition if treated efficiently in the early stages without the need for excision of the swelling.

\section{References}

1. Grove DI (2000) Tissue nematodes. In: Mandell GL, et al. (Eds.), Principles and practice of infectious diseases, $5^{\text {th }}$ (Edn.), London, England: Churchill Livingstone, pp: 246247.

2. Sabesan S, Palaniyandi M, Das PK, Michael E (2000) Mapping of lymphatic filariasis in India. Ann Trop Med Parasitol 94(6): 591-606.

3. Manguin S, Bangs MJ, Pothikasikorn J, Chareonviriyaphap $\mathrm{T}$ (2010) Review on global co-transmission of human Plasmodium species and Wuchereria Bancrofti by Anopheles mosquitoes. Infect Genet Evol 10(2): 159177.

4. Sharma P, Kumar N, Jain P, Gur R, Jain S (2005) Chronic Wuchereriasis presenting as a Vaginoperineal Fistula: Report of a Case with Aspiration Cytologic Diagnosis. Acta Cytol 49(3): 335-338.

5. Jain S, Sodhani P, Gupta S, Sakhuja P, Kumar N (2001) Cytomorphology of filariasis revisited: expansion of the morphologic spectrum and coexistence with other lesions. Acta Cytol 45(2): 186-191.

6. Michael E, Bundy DA, Grenfell BT (1996) Re-assessing the global prevalence and distribution of lymphatic filariasis, Parasitology 112(4): 409-428.

7. Park JE, Park K (2002) Park's textbook of preventive and social medicine, $18^{\text {th }}$ (Edn.), Jabalpur, India: Banarasidas Bhanot pp: 211-216.

8. Arora VK, Singh N, Bhatia A (1996) Cytomorphologic profile of lymphatic filariasis, Acta Cytol 40(5): 948-952.

9. Freedman DO, de Almeida A, Miranda J, Plier DA, Braga C (1997) Field trial of a rapid card test for Wuchereriabancrofti, Lancet 350(9092): 1681.

10. Ramzy RM, Farid HA, Kamal IH, Ibrahim GH, Morsy ZS, et al. (1997) A Polymerase chain reaction-based assay for detection of Wuchereria bancrofti in human blood and Culex pipiens. Trans R Sco Trop Med Hyg 91(2): 156-160.

11. Addiss DG, Beach MJ, Streit TG, Lutwick S, LeConte FH, et al. (1997) Randomised placebo- controlled comparison of ivermectin and albendazole alone and in combination for Wuchereria bancrofti micrifilaremia in Haitian children. Lancet 350(9076): 480-484. 